\title{
USO DEL JOB TO BE DONE COMO FUENTE DE DESARROLLO DE ESTRATEGIAS DE MARKETING DURANTE MOMENTOS DE CRISIS
}

\author{
USE OF JOB TO BE DONE AS A SOURCE FOR DEVELOPING MARKETING \\ STRATEGIES DURING TIMES OF CRISIS
}

Aniela García-Antonio ${ }^{1 *}$ (D); Ahtziri Lucero Beltrán-Sagundo ${ }^{2}$ (iD; Ana Laura Luna-Jiménez ${ }^{3}$ (D) .

1. Universidad Juárez Autónoma de Tabasco. aniela.garcia@ujat.mx

2. Universidad Juárez Autónoma de Tabasco. ahtziri2110@gmail.com

3. Universidad Juárez Autónoma de Tabasco. ana.luna.ujat@gmail.com

*Autor de Correspondencia: Aniela García-Antonio, correo electrónico: aniela.garcia@ujat.mx

\section{RESUMEN}

Comprender por qué el cliente adquiere un producto o servicio, tiene un nuevo protagonismo en el contexto de pandemia por Covid-19. La metodología Job to be Done propone detectar la razón por la que el consumidor contrata un producto o servicio, y de aquí emana la posibilidad de generar una solución efectiva para el cliente. Con este proyecto se busca detectar el trabajo que quiere hacer la persona que pide comida para llevar en la empresa familiar "Súper Tortas SagundoMR" ubicada en Tenosique, Tabasco; y de este modo construir estrategias enfocadas a dicho JTBD. Para ello, se realizó investigación cualitativa y cuantitativa con alcance transversal, a través de entrevistas y observación, así como el uso de la metodología Job Prioritization (Silverstein y De Carlo, 2012). Se encontró que esta empresa tiene potencial validado por sus consumidores en el servicio a domicilio y que sus razones para contratar este servicio se fincan en el sabor del producto. Existe potencial para reestablecer el servicio a domicilio utilizando el empuje que el valor de marca ha representado durante los últimos 30 años y de la validación del JTBD ganador.

Palabras clave: Pandemia; trabajos; servicio; insights.

Cómo citar:

García-Antonio, Aniela; Beltrán-Sagundo, Ahtziri Lucero; Luna-Jiménez, Ana Laura. (2021). USO DEL JOB TO BE DONE COMO FUENTE DE DESARROLLO DE ESTRATEGIAS DE MARKETING DURANTE MOMENTOS DE CRISIS. Revista de Investigaciones Universidad del Quindio, 33(S1), 32-42. https://doi.org/10.33975/riuq.vol33nS1.481 


\begin{abstract}
Understanding why the customer acquires a product or service has a new role in the context of the Covid-19 pandemic. The Job to be Done methodology proposes to detect the reason why the consumer hires a product or service, and from here emanates the possibility of generating an effective solution for the client. This project seeks to detect the work that the person who orders take-out food wants to do in the family business "Super Tortas SagundoMR" located in Tenosique, Tabasco; and in this way build strategies focused on said JTBD. For this, qualitative and quantitative research was carried out with a cross-sectional scope, through interviews and observation, as well as the use of the Job Prioritization methodology (Silverstein and De Carlo, 2012). It was found that this company has potential validated by its consumers in home delivery and that their reasons for hiring this service are based on the taste of the product. There is potential to re-establish home service using the push that brand equity has represented over the past 30 years and the validation of the winning JTBD.
\end{abstract}

Keywords: Pandemic; jobs; service; insights.

\title{
INTRODUCCIÓN
}

En momentos de crisis, entender las razones por las que un cliente consume un producto es un dato crucial para manejar una estrategia de marketing que mantenga a flote al negocio. Durante años se ha perseguido el entendimiento del cliente desde diversas metodologías con el objetivo de tener más certeza en las estrategias y acciones a desarrollar para obtener al final, mayores ingresos o una mejor posición en la industria. Durante la pandemia por Covid-19, este procedimiento puede ser la clave a la sobrevivencia del negocio.

Por un lado, Osterwalder \& Pigneur (2010), mencionan que la propuesta de valor es el factor que hace que un cliente se decante por una $u$ otra empresa. La finalidad es solucionar un problema o satisfacer una necesidad del cliente. Esta propuesta de valor puede ser innovadora y presentar una oferta diferente, mientras que otras pueden ser parecidas a ofertas ya existentes e incluir alguna característica o atributo adicional. Asimismo, la propuesta de valor tiene como finalidad solucionar problemas o satisfacer las necesidades de un cliente, e incluso constituye una serie de ventajas novedosas y que se pueden relacionar con la tecnología para satisfacer sus necesidades inexistentes (Díaz, Pérez, Hernández y Casto, 2017).

Por otra parte, Ulwick (2016), menciona que para asegurar una propuesta de valor ganadora, una empresa debe saber en qué parte del trabajo los clientes están desatendidos, qué propuesta comunican a los clientes donde sus necesidades puedan ser satisfechas y hacer todo lo que está en su poder para satisfacerlas; siendo estas mejor que sus competidores. Es así como las necesidades insatisfechas de hoy en día de los clientes, representan el valor ganador para muchas de las empresas y emprendedores.

Existe además, la metodología Job To Be Done (JTBD), la cual se centra no solo en la identificación de un producto, servicio o una solución específica, sino que busca conocer el propósito más elevado por el cual los clientes compran productos, servicios y soluciones; además que muestra el proceso por el cual un consumidor atraviesa cuando quiere transformar su situación o vida actual hacia algo mejor, pero no puede ya que hay barreras que los detiene (Silverstein, Philip y De Carlo, 2012). Esto ayuda a 
localizar, organizar y solucionar las necesidades emocionales, sociales, funcionales no satisfechas de los clientes; así como a descubrir las intenciones de los clientes al comprar un producto o servicio, ya que los clientes compran normalmente soluciones de una tarea que desean realizar.

Si se pudieran conocer las necesidades no satisfechas de los clientes, se podría llegar a manufacturar ideas que generen valor apegadas a la solución de sus problemas; conocer estos problemas podría llegar a cambiar la forma en que una empresa aborda el proceso de innovación, ya que con un conjunto de insights del cliente, una empresa puede descubrir oportunidades, entendiendo las situaciones desatendidas y atendidas, y con ello, decidir qué estrategias seguir. El JTBD, por ejemplo, es una metodología que lleva a las empresas a un mayor enfoque hacia sus clientes, descubriendo necesidades importantes a menudo (Ulwick, 2016). El propósito de esta investigación es centrarse en los trabajos del cliente cuando este quiere resolver un problema a través de un producto o servicio en específico. En la búsqueda de opciones de solución a un problema, en ocasiones los negocios asumen que conocen las razones de compra de un cliente, cuando es posible que éstas sean distintas o hayan cambiado a través del tiempo.

Un ejemplo de ello es el ya conocido tema de las malteadas de McDonalds (Chrystensen, Johnson y Rigby, 2002), en donde se dieron cuenta que este producto era un gran snack para comenzar el día, y con el cual podían tener algo en el estómago más sustancioso que un chocolate o un bagel (además de la eficiencia para ser ingerido mientras se manejaba hacia la oficina). Para dicho caso, la solución para mejorar las ventas no estaba en cambiar los sabores o hacerla más saludable; era acompañar a la persona en el camino a su trabajo. El cliente es consciente del contenido calórico del producto; y aun sabiendo ello lo compraban. No influía en su decisión de compra.

Presentamos en este trabajo una postura positiva hacia el profundo análisis del problema que el cliente quiere resolver, ya que al hacer esto desde el comienzo, permitiría mayor certeza de las estrategias de marketing propuestas, comunicando entonces información más cercana al usuario y cliente, esto logrado solo al haber observado detenidamente la situación del consumidor.

\section{MARCO TEÓRICO}

El ejercicio de entender lo que cliente busca en un producto ha sido un trabajo constante e ilimitado. Como mencionan Kotler y Armstrong (2016), el objetivo es encontrar un producto adecuado al cliente, por lo tanto, fundamentar las decisiones con datos, no había sido tan necesario como ahora.

A partir de la pandemia el mundo de los negocios cambió y las prioridades de los clientes y usuarios lo hicieron también. En México la situación económica ha provocado numerosos retos: por un lado, se estima que 420 millones de personas caigan en pobreza extrema, los sectores con mayores problemas son los que se encuentran en actividades secundarias, manufactureras y de construcción; y a largo plazo las MiPyMES serán las que tengan mayores repercusiones (Baker y Hernández, 2020). Bajo este contexto, los directivos de las empresas están enfocando sus esfuerzos para 2021 en entender al cliente, siendo la digitalización y la experiencia del cliente las prioridades para este año (Esquivel, 2021).

Con el propósito de conocer aquello que mueve al cliente a comprar, desde el estudio del marketing se han desarrollado nuevas maneras para obtener insights, que van desde la investigación de mercados (Malhotra, 2008), o el marketing basado en datos (Goyzueta, 2015). En paralelo, también se han 
desarrollado algunas metodologías generalmente basadas en el método científico donde a partir de una hipótesis, esta sea validada por el cliente potencial para tener mayor certeza de que el producto tenga la forma de la solución que estos buscaban.

Como se puede apreciar en la figura 1 notamos que metodologías como el Golden Ring (Sinek, 2009), Lean Startup (Rice, 2011), el Design Thinking (Brown, 2008), el Lienzo de la Propuesta de Valor (Osterwalder et.al, 2017), así como el Job to be Done (Chrysthensen et.al 2007) son algunas de las cuales han fincado su desarrollo en empatizar, observar y estudiar a la persona que consume un producto o servicio, y a través del uso de dicha técnica, se encuentre una solución con la que coincida en valor y por lo tanto la adquiera.

Figura 1. Metodologías para detectar problemas de los clientes

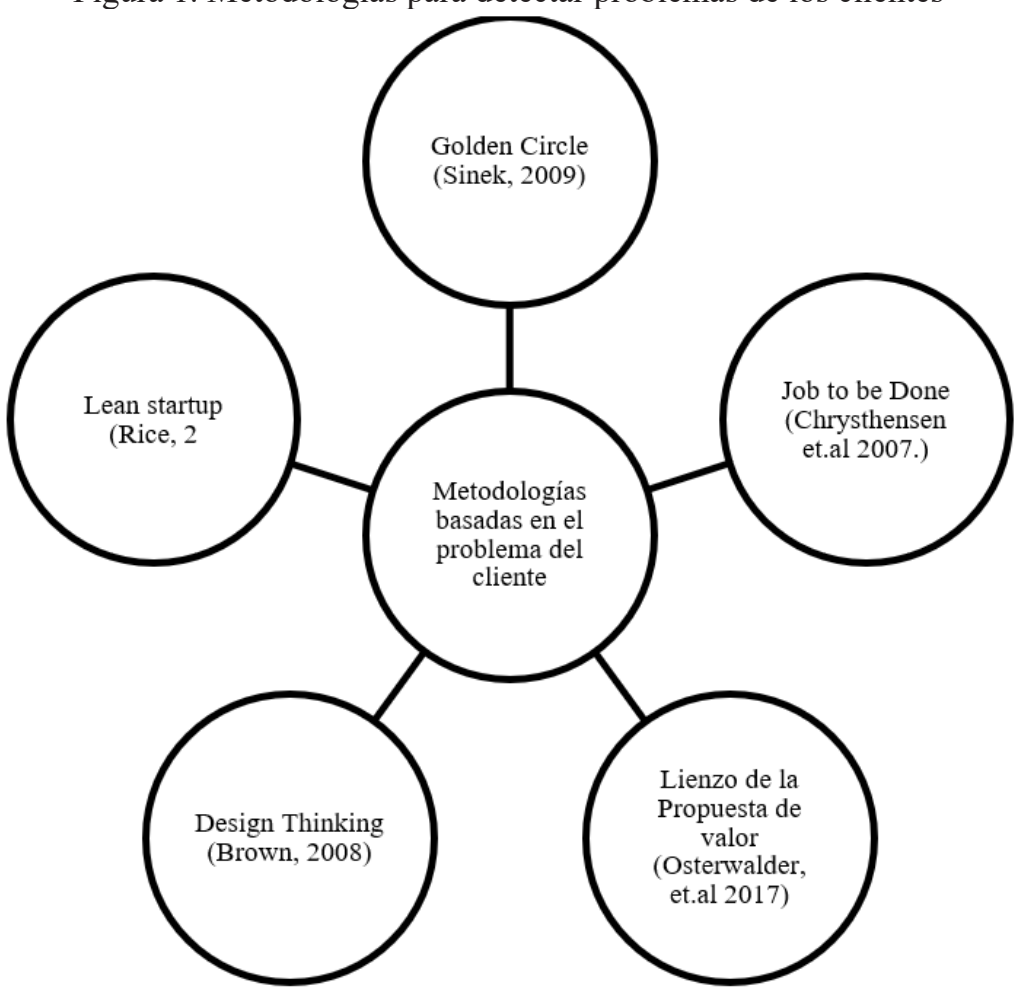

Fuente: elaboración propia

Deloitte (2020) menciona que las empresas que tienen un propósito son las que sortearán con más amabilidad los efectos de la pandemia. Para la firma consultora, una empresa con propósito es aquella que tiene una comprensión profunda de por qué existe la organización, sabiendo a quién está sirviendo y asegurándose que lo que ofrece es lo que este realmente busca. Recordando la metodología del Golden Circle -círculo dorado -, donde Simon Sinek (2009) menciona que el "por qué” de una empresa son las creencias y propósito de la misma, así como decir que "las personas no compran lo que haces, si no por qué lo haces", son las razones por las que comprender el propósito como empresa tiene una conexión crucial con el crecimiento de la misma. Sin embargo, si bien el propósito es importante, es necesario comprender al cliente para que así se haga un encaje del propósito empresarial y el trabajo del cliente.

Por otra parte se encuentra la metodología Design Thinking, la cual de acuerdo a Tim Brown (2008) es desarrollar innovación centrada en el cliente, a través del ejercicio de la empatía y la observación directa del viaje del consumidor encontrando una solución al problema o no encontrándolo, para que 
el pensador en diseño logre hallar una respuesta a través de lo que el mismo cliente ha ofrecido como pistas a la solución de su problema. Atinadamente la iteración y la observación son herramientas constantemente utilizadas durante un proceso de pensamiento en diseño, debido a la inmersión que se espera tener para entender al consumidor.

Con base en desarrollar una solución que pueda permanecer en el tiempo, nace también el lienzo de la propuesta de valor, desarrollado por Alexander Osterwalder et.al (2017) la cual es una herramienta basada en design thinking y el Job to be Done donde se busca ordenar la investigación hecha del cliente con base en lo que este quisiera para después ser nuevamente analizada con la intención de lograr una propuesta de valor ganadora apegada a lo que el usuario está buscando. Al igual que en el desarrollo del pensamiento en diseño, el proceso es iterativo hasta que se logra el encaje producto mercado. Al igual que en las demás metodologías revisadas, el centro de todo, es el cliente.

El método Lean Startup (Rice, 2012) últimamente utilizado en educación en emprendimiento es una robusta metodología que persigue en breves palabras, fallar menos, fallar rápido, reaprender y encontrar la mejor solución para una idea concebida. Quizás aunque no necesariamente se centre de origen en el cliente, sí es este quien validará la idea al final. De acuerdo con Rice (2012), "las startups todavía no saben quiénes serán sus consumidores o cuáles serán sus productos”. Bajo este tenor, podríamos decir que utilizando el lean startup estaríamos construyendo en paralelo un producto para un mercado por descubrir, inclusive hasta inexistente; lo cual es una ventaja si se considera que la inversión en dicho recorrido es menor comparándolo con una empresa con años en la insdutria.

Finalmente, el Job to be Done es una metodología que busca en las profundidades del pensamiento humano las razones por las que contratan un producto, para entender ese trabajo se desarrollan varias actividades alrededor. Se dice que las compañías se han dado cuenta que los clientes adquieren los productos para usarlo de manera diferente a la que originalmente tenía; de ahí la aseveración que para encontrar los trabajos del cliente se requiera observación, participación y menos uso de datos estadísticos, puesto que los trabajos se descubren en el movimiento de la vida (Chrysthensen, Anthony, Berstell y Nitterhouse, 2008).

Anthony Ulwrik (2018) menciona que "la teoría de los Jobs to be Done ofrece un marco para categorizar, definir, capturar y organizar todas las necesidades del cliente, y vincular métricas de rendimiento definidas por el cliente (en la forma de oraciones de resultados deseados)." Estos trabajos son aquellos que después se categorizan, se les da una prioridad, de acuerdo con la técnica que se utilice para ello, y posteriormente al análisis, este enunciado del trabajo se pueda utilizar como el valor más profundo por la que el cliente adquiere el producto. Es ahí donde, el Job to be Done puede ser utilizado como eje para el desarrollo de las estrategias de marketing. Esto se propone así considerando que no es un producto nuevo que se va a desarrollar, si no, es un producto existente pero donde no se le ha dado a conocer la razón por la que el cliente adquiere. Buscamos con este proyecto darle utilidad a esta metodología en otros ámbitos adicionales al de la creación de innovación, en este caso en el de las estrategias de marketing.

Hablando ahora de las estrategias de marketing, sabemos que se basan en una propuesta de valor que en la actualidad se debería fincarse en el problema que le resuelve al cliente. Como indica Kotler y Armstrong (2016) la propuesta de valor es un conjunto de beneficios o valores que promete entregar a los consumidores para satisfacer sus necesidades. Sin embargo sin embargo el principal reto en la creación de una estrategia ganadora, esta en que la propuesta de valor tenga como cimiento lo que al 
cliente le encantaría que su producto o servicio le resolviera. Aun así, prometer que un producto va a resolvernos un problema puede ser contraproducente. De acuerdo con Simon - Kucher \& Partners (2014) identificó que $72 \%$ de los productos nuevos fracasan, mencionando que la razón de esto es porque no se involucra al cliente en el proceso de innovación del producto o servicio. Asimismo, Ulrich y Eppinger (2013) agregan a esta reflexión, que para el desarrollo de productos es necesario que los clientes se involucren el proceso haciendo equipo con los colaboradores que desarrollan dichas soluciones para ellos.

Si consideramos que la propuesta de valor se puede considerar como una promesa, Seth Godin (2019, p.33) menciona que "la promesa está directamente relacionada con el cambio que quiere llevar a cabo, y está dirigida a la gente a la que se quiere cambiar”. Lo que nos puede llevar a una nueva reflexión: en el marketing ¿queremos cambiar a la gente? ó, ¿queremos entender al cliente?. Actualmente pareciera que hay una disyuntiva entre lo que el marketing hace y lo que el desarrollo de productos busca generar, máxime desde la perspectiva de los emprendimientos, donde la premisa original es ante todo la comprensión del cliente y sus problemas que de algún modo quieren resolver (Maurya, 2012; Chrysthensen et.al 2007, Ries, 2011; Brown, 2008).

Bajo esta consideración, el desarrollo de estrategias de marketing, que si bien se sabe, no son una apuesta segura (Kotler, Kartajawa y Setiawan, 2021), podría fallar con más seguridad si no se comprende a profundidad la razón por la que un cliente adquiere, máxime si dichas estrategias se crean sin hacer un claro análisis del problema que resuelve o el trabajo que contrata de ese producto o servicio. Si bien el marketing orientado al cliente (Kotler y Armstrong, 2016, p.11) menciona que "funciona bien cuando existe una necesidad clara y cuando los clientes saben lo que quieren", es una realidad que si los productos nuevos fallan, no solo es por una estrategia de marketing desalineada, también tiene que ver con la información que obtenemos del cliente y cómo la obtenemos. Y a partir de ello se han desarrollado distintos tipos de metodologías con el único propósito de fallar menos, de ayudar más al cliente, de proponer valor. Algunas de ellas son sumamente utilizadas en temas de emprendimiento, otras en el desarrollo de innovaciones y ello no excluye al marketing, toda vez que esta disciplina está íntimamente involucrada en la creación de nuevos productos y servicios, que al final le aporta a la empresa las estrategias para atraer al cliente y este resuelva su problema a través del producto promovido.

En este proyecto se propone el uso de Job to be Done como una metodología para la detección del trabajo que el cliente quiere resolver. Dentro de esta metodología, se encuentra el llamado Job Prioritization (Silverstein, Philip y De Carlo, 2012), el cual es parte de una robusta búsqueda del trabajo que el cliente quiere hacer al adquirir o contratar un producto o servicio. Este método consta de cinco pasos que ayuda a los emprendedores a saber las necesidades no satisfechas de sus clientes.

- Primer paso: identificar el mercado ya sea elaborando un nuevo producto, atendiendo las expectativas no atendidas de un trabajo, integrando las necesidades satisfechas individualmente o recreando el producto o servicio.

- Segundo paso: usar herramientas que ayuden a identificar los trabajos que los clientes quieren realiza.

- Tercer paso: enlistar las tareas

- Cuarto paso: declarar las tareas identificadas

- Quinto paso: se aplica el JTBD donde se elabora una gráfica para identificar las tareas aún no satisfechas o no realizadas, ya que estas son las que nos dan la oportunidad de innovar 
A partir del desarrollo de esta técnica se busca detectar el problema de un consumidor que pide comida para llevar en Súper Tortas Sagundo ${ }^{\mathrm{MR}}$ a través de la metodología JTBD; considerando como objetivos específicos' analizar el trabajo que el cliente hace cuando contrata un producto o servicio en Súper Tortas Sagundo ${ }^{\mathrm{MR}}$; así como desarrollar estrategias enfocadas en el JTBD detectado y las condiciones de vida a propósito de la COVID -19 en Tenosique Tabasco.

\section{MATERIALES Y MÉTODOS}

Los métodos utilizados para la realización de esta investigación están basados en la metodología presentada por Silverstein, Philip y De Carlo (2012) de los cuales se utilizaron el Job Statement y el Job Prioritization para la detección del JTBD ganador. Asimismo, se realizó investigación cualitativa y cuantitativa de alcance transversal, utilizando observación, entrevistas a dueños y consumidores, y encuesta a través de muestreo por conveniencia.

De acuerdo a Prieto (2013), el muestreo por conveniencia es una técnica no probabilística utilizada donde se encuestan personas que estuvieron en el momento y el lugar del proceso de investigación. Para nuestro caso, la pandemia por la Covid-19 no permitió por un lado la interacción directa con el cliente por cuestiones de confinamiento, y por otro lado, se buscaba entender el trabajo específico del cliente que adquiere productos para llevar, los cuales fueron encontrados vía telefónica y aleatoriamente se tomaron las llamadas de 30 personas con lo cual se lograron resultados que pueden dar un primer panorama de lo que actualmente emite la voz del cliente.

\section{HALLAZGOS}

El proyecto se realizó dentro de la empresa Súper Tortas Sagundo ${ }^{\mathrm{MR}}$, el cual es una pequeña empresa ubicada en Tenosique y en Centro, Tabasco, México. Comenzó hace 51 años vendiendo antojitos hechos con carne de pavo y pollo. En los últimos 10 años, su menú se ha basado en panuchos, caldos, tortas y sándwiches, todos elaborados con una receta familiar de pollo, la cual tiene influencias de la comida yucateca. Decidieron registrar su marca hace 30 años, y se han basado en un menú sencillo y seguro. Durante el confinamiento provocado por la pandemia de la Covid-19, decidieron retomar el servicio a domicilio, el cual dejaron de ofrecer hacía más de 20 años debido a inconvenientes con los clientes, ya que tuvieron llamadas de pedidos falsos en múltiples ocasiones y ello detonó la cancelación de este proceso. Desde el cierre en Marzo de 2020 debido a los efectos causados por la Covid-19, comenzaron a tener ventas bajas y hasta nulas; de acuerdo a entrevistas realizadas a los dueños, hubieron días donde solamente lograban obtener 100 pesos en ventas sin haber tomado al servicio a domicilio como opción para operar. Actualmente el negocio cuenta con tres sucursales, dos en Tenosique y uno, recién abierto en 2020 en Villahermosa, capital del estado de Tabasco; en este último,

Ahora, como parte del regreso del servicio a domicilio, y con la intención de entender las razones por las que un cliente pide comida para llevar se ha desarrollado una priorización de los Jobs to be Done; asimismo, a través de dicha comprensión, se desarrollarán estrategias específicas para los clientes solicitantes de este servicio durante y después del confinamiento.

Para aplicar el concepto del Job to be Done, se llevó a cabo el paso a paso de la metodología propuesta por Silverstein, Philip y De Carlo (2012), la cual sugiere se realice en cinco pasos, los cuáles son: 
1. Identifique un mercado de enfoque

2. Identifique los trabajos que los clientes intentan hacer

3. Crear declaraciones de trabajo

4. Priorice las oportunidades de JTBD

5. Aplicación del JTBD en la gráfica de Job Prioritization

De este modo, se espera obtener una declaración de trabajo clara que nos lleve a desarrollar una serie de estrategias focalizadas a lo que necesita resolver el cliente. A continuación se presentan los resultados emanados del uso de dicha metodología.

1. Identifique un mercado de enfoque. Para responder a esta cuestión, se realizó una entrevista a los dueños del negocio para conocer lo que ellos consideran que es su cliente. Cabe señalar que debido a la situación de pandemia, no se pudo observar físicamente quiénes son los integrantes del mercado; sin embargo, para hacer efectivo este proyecto, se consideró destacar al grupo de personas hombres y mujeres que adquieres sus alimentos a través de servicio a domicilio.

2. Identifique los trabajos que los clientes intentan hacer. Se realizó un sondeo a los clientes que llamaban para pedir servicio a domicilio, de las cuales, al preguntarles, su razón de compra, mencionaban en general que "pedían porque están deliciosos y bien preparados los antojitos", "por antojo" o "porque a su familia o hijos le gusta mucho" Clasifique los trabajos a realizar. De los resultados encontrados del sondeo consideramos la compra de antojitos en Súper Tortas Sagundo.

3. Crear declaraciones de trabajo. Se consideraron 12 JTBD, desarrollados con base en la obtención de datos previamente obtenidos en el sondeo, entrevista a dueña de la empresa y experiencia del encuestador; por ello, a continuación, se presentan los siguientes trabajos estructurados de acuerdo con la metodología del Job Statement (Silverstein y De Carlo, 2012):

1. Quiero comer antojitos sin tener que prepararlos

2. Quiero comer algo rico sin ir a un restaurante

3. Quiero comprar comida para mis familiares

4. Quiero cenar una torta porque se me antojó

5. Quiero solucionar la comida cuando no puedo cocinar

6. Quiero almorzar tortas en familia

7. Quiero regalar antojitos típicos a mis amigos

8. Quiero invitar a comer a alguien querido

9. Quiero probar nuevos antojitos

10. Quiero comprar consomé no hice comida en casa

11. Quiero pedir comida para comer en mi trabajo

12. Quiero comer panuchos en el lugar donde los preparan

4. Priorice las oportunidades de JTBD. Se realizó un sondeo a 30 personas sobre la importancia y la satisfacción de la empresa Súper Tortas Sagundo y encontramos que los trabajos 2,8,11 y 12 de acuerdo con la tabla 1, fueron los más altos en puntajes, por lo que se considera en ellos oportunidades para crear un espacio de mercado. 
Tabla 1. Priorización de los JTBD. Elaboración propia a través de la metodología Job Prioritization (Silverstein y DeCarlo, 2012).

\begin{tabular}{|l|c|c|c|}
\hline \multicolumn{1}{|c|}{ Job } & Importancia & Satisfacción & Oportunidad \\
\hline Job 1 Quiero comer antojitos sin tener que prepararlos & 4,3 & 2,0 & 6,7 \\
\hline Job 2 Quiero comer algo rico sin ir a un restaurante & 5,7 & 2,0 & 9,3 \\
\hline Job 3 Quiero comprar comida para mis familiares & 4,0 & 2,0 & 6,0 \\
\hline Job 4 Quiero cenar una torta porque se me antojó & 4,3 & 2,0 & 6,7 \\
\hline $\begin{array}{l}\text { Job 5 Quiero solucionar la comida cuando no puedo } \\
\text { cocinar }\end{array}$ & 4,3 & 2,0 & 6,7 \\
\hline Job 6 Quiero almorzar tortas en familia & 4,3 & 2,0 & 6,6 \\
\hline Job 7 Quiero regalar antojitos típicos a mis amigos & 3,7 & 2,0 & 5,3 \\
\hline Job 8 Quiero invitar a comer a alguien querido & 5,3 & 2,0 & 8,6 \\
\hline Job 9 Quiero probar nuevos antojitos & 4,3 & 2,0 & 6,6 \\
\hline $\begin{array}{l}\text { Job 10 Quiero comprar consomé no hice comida en } \\
\text { casa }\end{array}$ & 3,3 & 2,0 & 4,6 \\
\hline Job 11 Quiero pedir comida para comer en mi trabajo & 5,0 & 2,0 & 8,0 \\
\hline $\begin{array}{l}\text { Job 12 Quiero comer panuchos en el lugar donde los } \\
\text { preparan }\end{array}$ & 4,7 & 2,0 & 7,3 \\
\hline
\end{tabular}

Asimismo, como parte de la fórmula, encontramos la gráfica de la priorización de los JTBD. Para ello, se analizan los niveles de oportunidad a partir de la satisfacción y la importancia percibida en cada JTBD analizado. De acuerdo a Silverstein y De Carlo (2012), cuando se está en la zona "served right", como se muestra en la figura 1, se recomienda valorar a los JTBD relacionados, es decir, a los trabajos que se aproximan al trabajo principal del negocio, que en este caso es ofrecer comida preparada.

Figura 1. Priorización de los JTBD. Elaboración con base en la metodología Job Prioritization

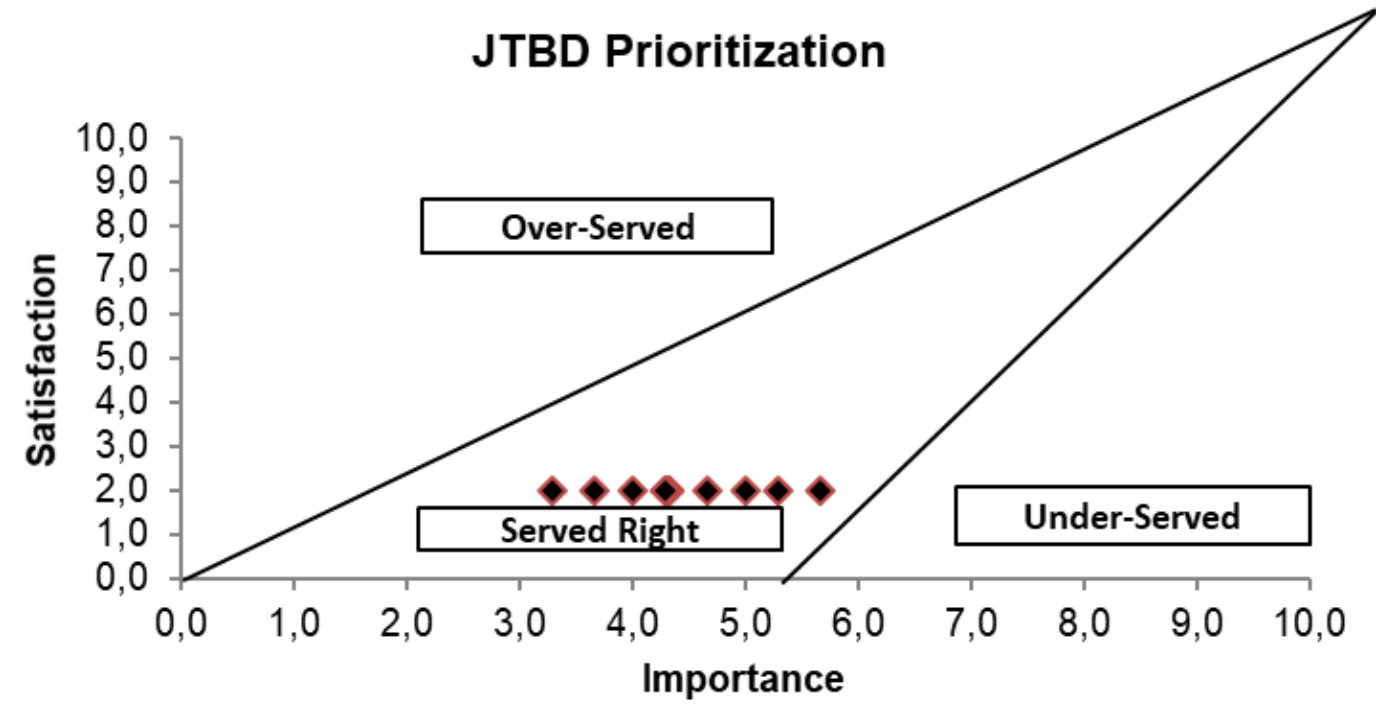

(Silverstein y De Carlo, 2012).

De este modo, los JTBD relacionados con la actividad principal y que obtuvieron mayor puntaje en la escala de "oportunidad", fueron: 
1. Job 2: Quiero comer algo rico sin ir a un restaurante con 9.3

2. Job 8: Quiero invitar a comer a alguien querido con 8.6

3. Job 11: Quiero pedir comida para comer en mi trabajo con 8.0

4. Job 12: Quiero comer panuchos en el lugar donde los preparan con 7.3

Con base en los resultados, se sugiere al negocio trabajar para elevar el valor del JTB 2, con lo cual potencie sus ofertas de servicio a domicilio a través de una mejor comercialización, tiempos y logística en la entrega del producto.

Las estrategias se han desarrollado bajo el método propuesto por Ferrell y Hartline (2012), las cuales tienen como base el JTBD 2 "Quiero comer algo rico sin ir a un restaurante". A continuación, se presentan:

\begin{tabular}{|l|l|}
\hline Meta & JTBD 2 "Quiero comer algo rico sin ir a un restaurante” \\
\hline Objetivo & $\begin{array}{l}\text { Incrementar 5\% las ventas a domicilio durante el primer } \\
\text { semestre de 2021. }\end{array}$ \\
\hline \multicolumn{1}{|c|}{ Estrategias } \\
\hline De distribución & $\begin{array}{l}\text { Desarrollar un protocolo sanitario donde el producto cumpla } \\
\text { con un protocolo de higiene y seguridad real y aparente a la } \\
\text { vista del consumidor final }\end{array}$ \\
\hline De publicidad & $\begin{array}{l}\text { Reposicionar la marca como una de sabor con historia que } \\
\text { encuentra nuevos caminos para llegar al consumidor usando } \\
\text { protocolos de cuidados sanitarios estandarizados. }\end{array}$ \\
\hline De promoción & Crear paquetes exclusivos para venta a domicilio. \\
\hline
\end{tabular}

\section{DISCUSIÓN Y CONCLUSIONES}

El Job to be Done es una metodología con la cual se pueden detectar razones por las que un cliente busca resolver un problema al comprar un producto o servicio específico. Al desarrollar el proceso de enunciados y priorización de este proceso, se puede visualizar que un cliente tiene el poder de declarar qué trabajo es el que quisieran que el producto resolviera, mientras que las áreas enfocadas en diseño de productos, puedan ilustrar la forma que quisieran que tuviera dicha solución. Si bien el cliente es capaz de decir lo que quiere, es una realidad que este es solo el primer paso para llegar a una solución clara y validad por ellos mismos.

Podemos notar que al usar el JTBD como meta de las estrategias de marketing, da al plan un camino con más certeza hacia lo que el cliente quiere contratar con cada producto o servicio a adquirir.

Para sus consumidores, Súper Tortas Sagundo ${ }^{\mathrm{MR}}$ tiene potencial para mejorar la propuesta de valor a través del servicio a domicilio; de acuerdo con el JTBD 2 "quiero comer algo rico sin ir a un restaurante", esto nos lleva a entender que el servicio a domicilio podría ser un aspecto que permanezca durante y después de la pandemia actual. Se sabe que el servicio a domicilio se volvió a utilizar debido a la situación de pandemia actualmente se vive, y hasta hoy ha tenido una aceptación suficiente para considerarlo como una idea de mejora. Se sugiere a la empresa mejorar las diferentes problemáticas encontradas luego de un análisis de los resultados como darle un buen ambiente a los establecimientos ya que algunos aun prefieren consumir en el local y dar una mejor comercialización, 
tiempos y logística en la entrega del producto. Súper Tortas Sagundo es un establecimiento que tiene más de 50 años, por lo que han logrado posicionar su prestigio en clientes reales y potenciales lo que le ha valido la recomendación de la marca. Al realizar un estudio como este, donde se priorice antes de tomar decisiones que signifiquen dinero a los trabajos que se deben resolver, se le da la oportunidad al negocio de poner sus esfuerzos centrados en lo que el cliente está esperando y no en lo que el empresario desea. Finalmente, quien adquiere el producto es aquel interesado en pagar por el mismo.

\section{REFERENCIAS}

1. Baker, J., \& Hernández, R. (2020). México después de la pandemia: Alternativas de reactivación económica. http://gcgenera.com/descargas/PolicyBrief_JuanCarlosBaker.pdf

2. Brown, T. (2008, septiembre). Design Thinking. Harvard Business Review América Latina. https:// emprendedoresupa.files.wordpress.com/2010/08/p02_brown-design-thinking.pdf

3. Christensen, C., Anthony, S. D., Berstell, G., \& Nitterhouse, D. (2007, 1 abril). Finding the Right Job For Your Product. MIT Sloan Management Review. https://sloanreview.mit.edu/article/ finding-the-right-job-for-your-product/

4. Christensen, C., Johnson, M., \& Rigby, D. (2002, 15 abril). Foundations for Growth: How to Identify and Build Disruptive New Businesses. MIT Sloan Management Review. https://sloanreview.mit. edu/article/foundations-for-growth-how-to-identify-and-build-disruptive-new-businesses/

5. Deloitte. (2021). 2021 Global Marketing Trends: find your focus. Deloitte Insights. https:// www2.deloitte.com/content/dam/Deloitte/cl/Documents/technology-media-telecommunications/ marketin-trends-2021/2021-GMT-Full-Report-CL.pdf

6. Díaz, A., Pérez, A., Hernández, J., \& Casto, M. (2017). Impacto de la cadena de valor en el margen de utilidad bruta en la producción de destilados de agave. Redalyc.org. https://www.redalyc.org/ articulo.oa? id $=14152127004$

7. Ferrell, O. C., \& Hartline, M. D. (2012). Estrategia de marketing (5a. ed.). Cengage Learning.

8. Godin, S. (2019). Esto es marketing. Alianza Editorial.

9. Goyzueta, S. (2015, mayo). Big Data Marketing: una aproximación. Perspectivas. http://www. scielo.org.bo/pdf/rp/n35/n36_a07.pdf

10. Herrera, J. E. P. (2013). Investigación de mercados. Ediciones Pirámide.

11. K. (2016). Marketing (16. ed.). Pearson Educación.

12. Esquivel, V. (2021). Panorama 2021: Cuatro prioridades en una nueva realidad. KPMG.

13. Kotler, P., Kartajaya, H., \& Setiawan, I. (2021). Marketing 5.0. Wiley.

14. Malhotra, N. (2008). Investigacion De Mercados (5.a ed.). Pearson Educación.

15. Maurya, A. (2012). Running Lean. Van Duuren Media.

16. Osterwalder, A., Pigneur, Y., Bernarda, G., Smith, A., \& Papadakos, T. (2014). Value Proposition Design. Wiley.

17. Ries, E., \& Julián, J. S. (2012). El método Lean Startup. Deusto.

18. Silverstein, D., DeCarlo, N. (2012). The Innovator's Toolkit. Wiley.

19. Simon - Kucher \& Partners. (2014). Global Pricing Study 2014.

20. Sinek, S. (2009). Start with why. Portfolio.

21. Ulrich, K., \& Eppinger, S. (2011). Product Design and Development (5th ed.). Irwin/McGrawHill.

22. Ulwick, A. W., \& Osterwalder, A. (2016). Jobs to Be Done. Amsterdam University Press. 